\title{
Soliton Dynamics in Linearly Coupled Discrete Nonlinear Schrödinger Equations
}

\author{
A. Trombettoni ${ }^{1}$, H.E. Nistazakis ${ }^{2}$, Z. Rapti ${ }^{3}$, D.J. Frantzeskakis ${ }^{2}$, and P.G. Kevrekidis ${ }^{4}$ \\ 1 International School for Advanced Studies and Sezione INFN, Via Beirut 2/4, I-34104, Trieste, Italy \\ 2 Department of Physics, University of Athens, Panepistimiopolis, Zografos, Athens 15784, Greece \\ ${ }^{3}$ Department of Mathematics, University of Illinois at Urbana-Champaign, Urbana, Illinois 61801-2975, USA \\ 4 Department of Mathematics and Statistics, University of Massachusetts, Amherst MA 01003-4515, USA
}

\begin{abstract}
We study soliton dynamics in a system of two linearly coupled discrete nonlinear Schrödinger equations, which describe the dynamics of a two-component Bose gas, coupled by an electromagnetic field, and confined in a strong optical lattice. When the nonlinear coupling strengths are equal, we use a unitary transformation to remove the linear coupling terms, and show that the existing soliton solutions oscillate from one species to the other. When the nonlinear coupling strengths are different, the soliton dynamics is numerically investigated and the findings are compared to the results of an effective two-mode model. The case of two linearly coupled Ablowitz-Ladik equations is also investigated.
\end{abstract}

\section{INTRODUCTION}

The study of discrete solitons is a central topic in nonlinear lattice dynamics [1, 2, 3]. Of particular relevance is the robustness of soliton motion against perturbations and the possibility of controlling the soliton dynamics. Accordingly, many recent studies on this subject are focused on multi-component nonlinear lattices (see, e.g., [4, 5] and references therein). In this context, the inter-component coupling has a crucial role, as it may either destabilize the soliton propagation, or it can be used as a resource to move the soliton from one component to the other in a controllable manner.

The interest in multi-component systems is considerably motivated by the experimental realization and control of mixtures of Bose-Einstein condensates (BECs) composed either by different hyperfine states [ 6, 7, 8] (including spinor BECs [9]), or by different species [10, 11]. Importantly, multi-component BECs can be trapped in strong optical lattices, as in the recent experiment reported in Ref. 12]. The optical lattice can be created by one or more pairs of counterpropagating laser beams, giving rise to a periodic potential in one, two, or three spatial dimensions [13]. In such a case, the dynamics of each (single-component) BEC is typically well described by a discrete dynamical model, namely the discrete nonlinear Schrödinger equation (DNLSE) 14] - see also [13, 15, 16] for reviews and references therein. Denoting by $\Psi_{j}$ the BEC wavefunction on site $j$ of the optical lattice, this model reads

$$
i \hbar \frac{\partial \Psi_{j}}{\partial t}=-K\left(\Psi_{j-1}+\Psi_{j+1}\right)+u\left|\Psi_{j}\right|^{2} \Psi_{j}+V_{j} \Psi_{j},
$$

where $K$ is the tunneling rate between neighboring sites, $u$ is the nonlinear coefficient proportional to the s-wave scattering length, and $V_{j}$ accounts for an external potential that may be superimposed to the lattice. Notice that although Eq. (1) has been written for a one-dimensional $(1 D)$ setting, a generalization to higher-dimensions is straightforward. The DNLSE is a typical example of a discrete dynamical system, whose properties have been intensively studied [3, 15, 17]. The interest for the DNLSE model arises from the fact that it has been successfully used to describe the dynamical properties of several systems (including, e.g., arrays of coupled optical waveguides [18]). In $1 D$, the DNLSE is not integrable [17]; however, and as concerns the homogeneous case $\left(V_{j}=0\right)$, solitonlike wavepackets exist and can propagate for a long time as stable objects, as it can be shown, e.g., by variational approaches $[19,20]$. Furthermore, the dynamics of such traveling pulses has been investigated in detail in the literature $21,22,23,24,25]$.

Based on the above discussion, here we consider the dynamics of a multicomponent BEC in an optical lattice described by a system of coupled DNLSEs, the different BEC components being different hyperfine levels [26]. Since the atoms of different components interact with each other, one has a nonlinear coupling between the different DNLSE; furthermore, one can induce a linear Rabi coupling by coupling the different hyperfine levels by an electromagnetic field [27, 28].

The prototypical system we consider is a two-component Bose gas in an external trapping potential: typically the condensates are different Zeeman levels of alkali atoms like ${ }^{87} \mathrm{Rb}$. Experiments with a two-component ${ }^{87} \mathrm{Rb}$ condensate use atom states customarily denoted by $|1\rangle$ and $|2\rangle$; in particular, the states can be $\left|F=2, m_{F}=1\right\rangle$ and $|2,2\rangle$, like, e.g., in [29], or $|1,-1\rangle$ and $|2,1\rangle$, like, e.g., in [30] (see also the recent work [8]). In general, the condensates $|1\rangle$ and $|2\rangle$ have different magnetic moments: then in a magnetic trap they can be subjected to different magnetic potentials, eventually centered at different positions and having the same frequencies (like in the setup described in [30]) or 
different frequencies [29]; in the latter work, the ratio of the frequencies of $V_{2}$ and $V_{1}$ is $\sqrt{2}$. It is also possible to add a periodic potential acting on the two-component Bose gas 26.

The two Zeeman states $|1\rangle$ and $|2\rangle$ can be coupled by an electromagnetic field with frequency $\omega_{\text {ext }}$ and strength characterized by the Rabi frequency $\Omega_{R}$. A discussion of (and references on) the experimental manipulation of multicomponent Bose gases are in [27, 28].

In the case of a binary BEC mixture confined in a one-dimensional lattice the system of the two coupled DNLSEs takes the form

$$
\begin{aligned}
& i \hbar \frac{\partial \psi_{(1) j}}{\partial t}=-K_{1}\left(\psi_{(1) j-1}+\psi_{(1) j+1}\right)+u_{11}\left|\psi_{(1) j}\right|^{2} \psi_{(1) j}+u_{12}\left|\psi_{(2) j}\right|^{2} \psi_{(1) j}+V_{j} \psi_{(1) j}+\Omega(t) \psi_{(2) j} \\
& i \hbar \frac{\partial \psi_{(2) j}}{\partial t}=-K_{2}\left(\psi_{(2) j-1}+\psi_{(2) j+1}\right)+u_{12}\left|\psi_{(1) j}\right|^{2} \psi_{(2) j}+u_{22}\left|\psi_{(2) j}\right|^{2} \psi_{(2) j}+V_{j} \psi_{(2) j}+\Omega(t) \psi_{(1) j}
\end{aligned}
$$

In Eqs. (2)-(3), $\psi_{(\alpha) j}$ denotes the wavefunction of the condensate $\alpha(\alpha=1,2)$ on site $j, \Omega(t)$ is proportional to the Rabi frequency (i.e., the strength of the electromagnetic field), the nonlinear coefficients $u_{\alpha \beta}$ are proportional to the scattering lengths $a_{\alpha \beta}$ for species $\alpha$ and species $\beta$, accounting for intra- $(\alpha=\beta)$ and inter- $(\alpha \neq \beta)$ species interactions. In Eqs. (2)-(3) we also assumed that the external potential superimposed to the optical lattice is equal for both species. Furthermore, $K_{\alpha}$ is the tunneling rate between nearest-neighbour sites for particles of the condensate $\alpha$; for a two-component condensate, when the periodic potential is the same for both the hyperfine levels one has $K_{1}=K_{2}$, otherwise one has $K_{1} \neq K_{2}[31]$.

In this work we are interested in studying the dynamics of the system, which is prepared with a soliton initially present in a single component, after the linear coupling $\Omega$ is turned on. Our presentation is organized as follows. First, in Section II we show that for $K_{1}=K_{2}$ it is possible to perform a unitary transformation to remove the linear coupling, analogous to the one valid for two linearly coupled continuous Gross-Pitaevskii equations [32] (see also earlier relevant work in the context of nonlinear optics [33, 34]); one can then use the results valid for a single-component DNLSE to determine the soliton dynamics. The case $K_{1} \neq K_{2}$ is numerically studied, and our findings are compared in Section III with the results of a simplified two-mode model. In Section IV, we consider two linearly coupled Ablowitz-Ladik equations (which is an integrable variant of the DNLSE [35]) showing results similar to those obtained for two coupled DNLSEs. Finally, in section V, we briefly summarize our findings and present some interesting directions for future work.

\section{EQUAL TUNNELING RATES}

\section{A. General Lattice}

In this Section we study the dynamics of a soliton initially present in one of the components, when the linear coupling with the other component is turned on. Although we are interested in solitons in $1 D$ coupled DNLSEs, to show the generality of our approach we write the Eqs. (2)-(3) on a general lattice as

$$
\begin{aligned}
& i \hbar \frac{\partial \psi_{(1) j}}{\partial t}=-K_{1} \sum_{n(j)} \psi_{(1) n}+u_{11}\left|\psi_{(1) j}\right|^{2} \psi_{(1) j}+u_{12}\left|\psi_{(2) j}\right|^{2} \psi_{(1) j}+V_{j} \psi_{(1) j}+\Omega(t) \psi_{(2) j}, \\
& i \hbar \frac{\partial \psi_{(2) j}}{\partial t}=-K_{2} \sum_{n(j)} \psi_{(2) n}+u_{12}\left|\psi_{(1) j}\right|^{2} \psi_{(2) j}+u_{22}\left|\psi_{(2) j}\right|^{2} \psi_{(2) j}+V_{j} \psi_{(2) j}+\Omega(t) \psi_{(1) j},
\end{aligned}
$$

where the sum $\sum_{n(j)}$ is on the neighbors $n$ of the site $j$. We will also consider equal interaction-strengths $\left(u_{11}=\right.$ $u_{22}=u_{12}=u$ ), as it is almost the case, e.g., for the two-component ${ }^{87}$ Rb BECs studied in [29, 30]; in fact, in Ref. [30], the ratios $u_{11}: u_{12}: u_{22}=0.97: 1.00: 1.03$ were used, while in Ref. [29] $u_{11}: u_{12}: u_{22}=1.00: 1.00: 0.97$.

When $K_{1}=K_{2}$ Eqs. (4)-(5) can be rewritten in a more compact form as:

$$
i \hbar \frac{\partial \psi_{j}}{\partial t}=\mathcal{T} \psi_{j}+\left(\psi_{j}^{\dagger} G \psi_{j}\right) \psi_{j}+V_{j} \psi_{j}+\Omega(t) P \psi_{j},
$$

where

$$
\psi_{j}=\left(\begin{array}{l}
\psi_{(1) j} \\
\psi_{(2) j}
\end{array}\right), \quad G=u\left(\begin{array}{ll}
1 & 0 \\
0 & 1
\end{array}\right), \quad P=\left(\begin{array}{ll}
0 & 1 \\
1 & 0
\end{array}\right)
$$


and

$$
\mathcal{T} \psi_{j} \equiv-K \sum_{n(j)} \psi_{n}
$$

Then, similarly to the case of the continuum counterpart of our model [32, 33, 34], we introduce the spinor field $\phi_{i}$ through the relation

$$
\psi_{i}=U(t) \phi_{i}
$$

where $U(t)$ is given by

$$
U(t)=\exp [-i P \mathcal{I}(t)]=\left(\begin{array}{cc}
\cos \mathcal{I}(t) & -i \sin \mathcal{I}(t) \\
-i \sin \mathcal{I}(t) & \cos \mathcal{I}(t)
\end{array}\right)
$$

with $\mathcal{I}(t)=(1 / \hbar) \int_{0}^{t} \Omega\left(t^{\prime}\right) d t^{\prime}$, and obtain,

$$
i \hbar \frac{\partial \phi_{j}}{\partial t}=\mathcal{T} \phi_{j}+\left(\phi_{j}^{\dagger} G \phi_{j}\right) \phi_{j}+V_{j} \phi_{j}
$$

which corresponds to Eq. (6) without the Rabi term proportional to $\Omega(t) P$. This transformation was originally introduced in Ref. [33] (see also 34]), where it was used to eliminate a constant linear coupling term by a change of polarization basis in a set of two coupled continuous NLS equations modeling optical pulse propagation in birefringent optical fibers, and later was used in the context of binary BECs in Ref. [32]. It is important to stress that the above transformation (91)-(10) eliminates the Rabi term only when $G$ and $P$ commute; in other words, the transformation is only possible in the so-called Manakov case [36], where all interaction strengths are equal, i.e., $u_{11}=u_{22}=u_{12}$. In the case of nonequal intra- and inter-species interaction strengths, i.e., $u_{11}=u_{22} \neq u_{12}$, the transformation can still be applied but the final equation contains a more involved nonlinear interaction term. This physically relevant situation was recently considered in Ref. [37], where the latter calculation was carried out for the continuous case.

Let us now consider a soliton solution of Eq. (6) with the the linear coupling turned off $(\Omega(t)=0)$ with initial condition

$$
\psi_{j}(t=0)=\left(\begin{array}{c}
\Psi_{j}(t=0) \\
0
\end{array}\right)
$$

i.e., all particles are initially in the first component. Here, $\Psi_{j}(t)$ denotes the soliton solution of the single component DNLSE for the condensate 1 , which we assume to be known. If at time $t=t_{0}$ the linear coupling is turned-on and the Rabi frequency has a general time dependence $\Omega(t)$, then it holds

$$
\psi_{j}\left(t_{0}\right)=\phi_{j}\left(t_{0}\right)=\left(\begin{array}{c}
\Psi_{j}\left(t_{0}\right) \\
0
\end{array}\right)
$$

Due to the fact that Eq. (11), which describes the evolution of $\phi$ for $t>t_{0}$, is identical to the one describing the evolution of $\psi$ for $t<t_{0}$ (i.e., without the Rabi coupling), it follows that for $t>t_{0}$ one has:

$$
\phi_{j}(t)=\left(\begin{array}{c}
\Psi_{j}(t) \\
0
\end{array}\right)
$$

Using Eq. (10), one gets

$$
\psi_{j}(t)=\left(\begin{array}{c}
\cos \mathcal{I}(t) \cdot \Psi_{j}(t) \\
-i \sin \mathcal{I}(t) \cdot \Psi_{j}(t)
\end{array}\right)
$$

Equation (15) shows that the soliton can tunnel without losing its shape, and the effect of the transformation is just a change in the normalization. Hence, normalizing $\Psi_{j}$ to 1 and denoting

$$
N_{\alpha}=\sum_{j}\left|\psi_{(\alpha) j}\right|^{2}
$$

the fraction of the number of particles in condensate $\alpha$, one gets $N_{1}=1$ and $N_{2}=0$ for $t \leq t_{0}$, as well as

$$
N_{1}=\cos ^{2} \mathcal{I}(t),
$$

and $N_{2}=1-N_{1}$ for $t>t_{0}$. We notice that the result (17) does not depend on the particular soliton solution chosen, nor on the momentum of the soliton. 


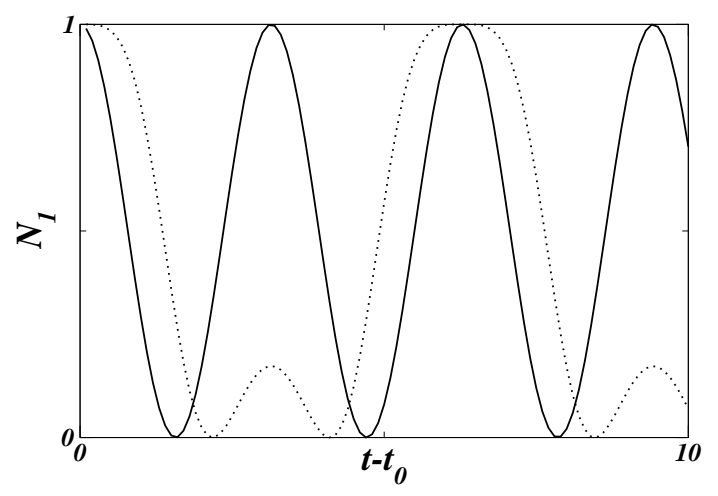

FIG. 1: Fraction of the number of particles $N_{1}$ in the condensate 1 as a function of time for $\Omega(t)=\Omega_{0}$ (solid line) and $\Omega(t)=\Omega_{0} \sin \beta\left(t-t_{0}\right)$ (dotted line), where $\beta=\Omega_{0} / \hbar$. In both cases, time is in units of $\Omega_{0} / \hbar$.

\section{B. 1D Chain}

As an example of the previous results we considered the 1D case, and in Fig. 1 we plot (for $t>t_{0}$ ) the number of particles $N_{1}$ in the first component, for a constant Rabi frequency $\Omega(t)=\Omega_{0}$ and for a sinusoidal Rabi frequency $\Omega(t)=\Omega_{0} \sin \beta\left(t-t_{0}\right)$ versus time. One can clearly see that in both cases $N_{1}$ oscillates between the values one and zero, as expected.

As a further application of the previous analysis we consider the $1 \mathrm{D}$ homogeneous problem with $V_{j}=0$. In this case, measuring time in units of $\hbar / 2 K_{1}$ and energies in units of $2 K_{1}$, the rescaled coupled DNLSEs read

$$
\begin{gathered}
i \frac{\partial \psi_{(1) j}}{\partial t}=-\frac{1}{2}\left(\psi_{(1) j+1}+\psi_{(1) j-1}\right)+\Lambda\left(\left|\psi_{(1) j}\right|^{2}+\left|\psi_{(2) j}\right|^{2}\right) \psi_{(1) j}+\omega(t) \psi_{(2) j}, \\
i \frac{\partial \psi_{(2) j}}{\partial t}=-\frac{1}{2} R\left(\psi_{(2) j+1}+\psi_{(2) j-1}\right)+\Lambda\left(\left|\psi_{(1) j}\right|^{2}+\left|\psi_{(2) j}\right|^{2}\right) \psi_{(2) j}+\omega(t) \psi_{(1) j},
\end{gathered}
$$

where $\Lambda=u / 2 K_{1}, \omega=\Omega / 2 K_{1}$ and $R=K_{2} / K_{1}$ is assumed to be equal to 1 . At $t=0$ all particles are supposed to be in the first component in a soliton-like wavepacket and $\omega=0$ for $t<t_{0}$. It is known that on a chain, the single-component DNLSE soliton-like solutions can propagate for a long time even if the equation is not integrable [3]. Therefore, we consider, at $t=0$, a gaussian wavepacket centered at $\xi(t=0) \equiv \xi_{0}$, with initial momentum $p_{0}$ and width $\gamma(t=0)=\gamma_{0} \equiv \sqrt{\alpha_{0}}$. Then, its temporal evolution can be analyzed by a variational approach, where we assume that the wavefunction has the following form

$$
\Psi_{n}^{V}(t)=\sqrt{\mathcal{K}} \cdot \exp \left[-\frac{(n-\xi)^{2}}{\alpha}+i p(n-\xi)+i \frac{\delta}{2}(n-\xi)^{2}\right]
$$

where $\xi(t)$ and $\gamma(t)=\sqrt{\alpha(t)}$ are the center and the width of the density respectively, having conjugate momenta $p(t)$ and $\delta(t)$ ( $\mathcal{K}$ is a normalization factor). Writing the equations of motion for $\xi, p, \alpha, \delta$ one obtains $p(t)=p_{0}$ and $\dot{\xi}=\sin p_{0} e^{-\eta}$, with $\eta=1 /(2 \alpha)+\alpha \delta^{2} / 8$. Imposing the conditions $\dot{\gamma}=0$ and $\dot{\delta}=0$, it follows that for $\cos p_{0}<0$ the value of $\Lambda$ (when $\left.\gamma_{0} \gg 1\right)$ for which a (variational) soliton solution exists is [14]:

$$
\Lambda_{\text {sol }}=2 \sqrt{\pi} \frac{\left|\cos p_{0}\right|}{\gamma_{0}}
$$

The stability of the variational solutions for large times has been investigated numerically in [19, 20]. For the value $\Lambda_{\text {sol }}$ given by Eq. (21) one has $\alpha(t)=\alpha_{0}, \delta(t)=0$ and $\dot{\xi}=\sin p_{0} \cdot e^{-1 /\left(2 \alpha_{0}\right)} \approx \sin p_{0}$. Notice that $p=\pi$ corresponds to a gap soliton.

If at $t=t_{0}$ we turn on the Rabi switch with frequency $\omega(t)$, then we obtain

$$
\psi_{j}(t)=\left(\begin{array}{c}
\cos \mathcal{I}(t) \cdot \Psi_{j}^{V}(t) \\
-i \sin \mathcal{I}(t) \cdot \Psi_{j}^{V}(t)
\end{array}\right)
$$






FIG. 2: Fraction of the number of particles $N_{1}$ in the condensate 1 as a function of time for $p=\pi$ (solid line) and $p=3 \pi / 4$ (dashed line) obtained from the numerical solution of the coupled DNLSEs (18)-(19) with the initial condition given by (20) and $\Lambda=\Lambda_{\text {sol }}$. The two lines are indistinguishable and coincide with (17), also plotted as a dotted line. We used the parameter values: $\Lambda=1, t_{0}=10, \omega=0.2, \gamma_{0}=50$.

The numerical solution of the coupled DNLSEs (18)-(19), with the initial condition $\psi_{(1) j}(t=0)=\Psi_{j}^{V}(t=0)$ given by (20) and $\Lambda=\Lambda_{\text {sol }}$, turns out to be in excellent agreement with (22) for different values of $p$, as shown in Fig. 2 for $\omega$ constant.

\section{DIFFERENT TUNNELING RATES}

When the tunneling rates are different (i.e., $R=K_{1} / K_{2} \neq 1$ ), the unitary transformation (10) does not really simplify the problem. Actually, Eqs. (4)-(5) can be written in the compact form (6) with the notation

$$
\mathcal{T} \psi_{j} \equiv \mathcal{T}_{K_{1} ; K_{2}} \psi_{j}=-\left(\begin{array}{c}
K_{1} \sum_{n(j)} \psi_{(1) n} \\
K_{2} \sum_{n(j)} \psi_{(2) n}
\end{array}\right)
$$

By performing the transformation (10) one finally gets

$$
i \hbar \frac{\partial \phi_{j}}{\partial t}=\mathcal{T}_{\tilde{K}_{1} ; \tilde{K}_{2}} \phi_{j}+\left(\phi_{j}^{\dagger} G \phi_{j}\right) \phi_{j}+V_{j} \phi_{j}-\sigma_{y} \Delta \mathcal{S}(t) \mathcal{C}(t) \sum_{n(j)} \phi_{j}
$$

where $\Delta=K_{1}-K_{2}, \mathcal{S}=\sin \mathcal{I}, \mathcal{C}=\cos \mathcal{I}, \tilde{K}_{1}(t)=K_{1}-\Delta \mathcal{S}^{2}(t), \tilde{K}_{2}(t)=K_{2}+\Delta \mathcal{S}^{2}(t)$, and $\sigma_{y}=\left(\begin{array}{cc}0 & -i \\ i & 0\end{array}\right)$. It therefore follows that the effect of the transformation (10) is to replace the general time dependence $\Omega(t)$ of the Rabi switch, by the last term of Eq. (24). So, whenever the tunneling rates are different we study Eqs. (44)-(5) directly.

In Fig. 3 we plot the time dependence of $N_{1}$ for different values of $R$. It is observed that $N_{1}$ does not reach the value zero, i.e., the soliton cannot be totally transferred to the other component. Moreover, it is clear that the larger the deviation of $R$ from 1 is, the larger the minimum reached value of $N_{1}$ becomes, and therefore the more inefficient the transferring process becomes.

These numerical results can be understood by means of a simple two-mode model, where for $t>t_{0}$ the variational wavefunction is assumed to be of the form

$$
\psi_{j}^{V}=\left(\begin{array}{c}
\psi_{(1) j}^{V} \\
\psi_{(2) j}^{V}
\end{array}\right)=\Psi_{j}^{V} \cdot\left(\begin{array}{c}
\sqrt{N_{1}(t)} e^{i \varphi_{1}(t)} \\
\sqrt{N_{2}(t)} e^{i \varphi_{2}(t)}
\end{array}\right) .
$$

Here, $\Psi_{j}^{V}$ is given by (20) and the variational parameters are now the center $\xi$, the momentum $p$, the width $\sqrt{\alpha}$ and its momentum $\delta$, plus $N_{1}, N_{2}$ (the number of particles in the two components) and the phases $\varphi_{1}, \varphi_{2}$. Note that a similar to Eq. (25) variational ansatz has been used in the past to study soliton dynamics in two linearly coupled continuous NLS equations describing pulse propagation in dual-core optical fibers [38]. 

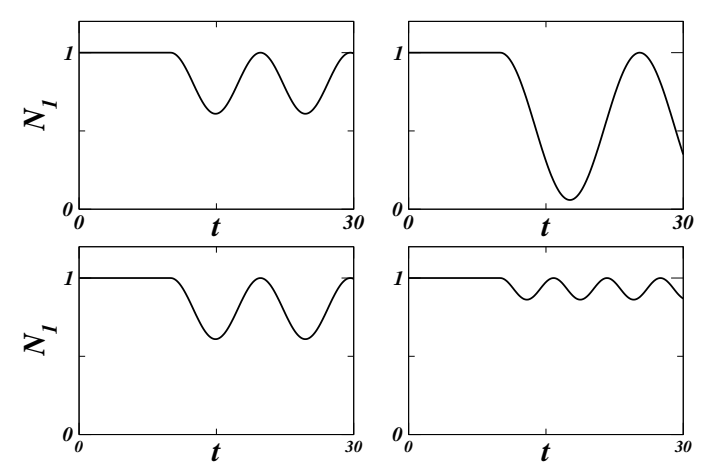

FIG. 3: Fraction of the number of particles $N_{1}$ in the first component as a function of time for $p=\pi$ obtained from the numerical solution of the coupled DNLSEs for four different values of $R$, namely 0.5 (top-left), 0.9 (top-right), 1.5 (bottom-left), and 2.0 (bottom-right); the rest of the parameters are the same as in Fig. 2

The Lagrangian $\mathcal{L}$ is given by

$$
\mathcal{L}=i \sum_{j} \psi_{j}^{V \dagger} \frac{\partial}{\partial t} \psi_{j}^{V}-\mathcal{H}
$$

where the Hamiltonian $\mathcal{H}$ is

$$
\begin{gathered}
\mathcal{H}=-\frac{1}{2} \sum_{j}\left(\psi_{(1) j} \psi_{(1) j+1}^{*}+\text { c.c. }\right)-\frac{R}{2} \sum_{j}\left(\psi_{(2) j} \psi_{(2) j+1}^{*}+\text { c.c. }\right) \\
+\omega \sum_{j}\left(\psi_{(1) j} \psi_{(2) j}^{*}+\text { c.c. }\right)+\frac{\Lambda}{2} \sum_{j}\left(\left|\psi_{(1) j}\right|^{4}+\left|\psi_{(2) j}\right|^{4}+2\left|\psi_{(1) j}\right|^{2}\left|\psi_{(2) j}\right|^{2}\right) .
\end{gathered}
$$

Omitting the details, the equations of motion for $p$ and $\xi$ are found to be

$$
\begin{aligned}
& \dot{p}=0, \\
& \dot{\xi}=\left(N_{1}+R N_{2}\right) \sin p \cdot e^{-\eta},
\end{aligned}
$$

(where $\left.\eta=1 /(2 \alpha)+\alpha \delta^{2} / 8\right)$, and those for $\alpha$ and $\delta$ are

$$
\begin{aligned}
\dot{\delta} & =\left(N_{1}+R N_{2}\right) \cos p \cdot\left(\frac{4}{\alpha^{2}}-\delta^{2}\right) e^{-\eta}+2 \Lambda / \sqrt{\pi} \gamma^{3}, \\
\dot{\alpha} & =2\left(N_{1}+R N_{2}\right) \alpha \delta \cos p \cdot e^{-\eta} .
\end{aligned}
$$

One sees that for $R=1$, since $N_{1}+N_{2}=1$, Eqs. (28)-(31) do not depend on the equations for $N_{1}, N_{2}, \varphi_{1}, \varphi_{2}$. When $R \neq 1$, the dynamics of the internal degrees of freedom is coupled with the phase-number dynamics. The equations for $N_{1}$ and $N_{2}$ are

$$
\begin{aligned}
& \dot{N}_{1}=2 \omega \sqrt{N_{1} N_{2}} \sin \varphi \\
& \dot{N}_{2}=-2 \omega \sqrt{N_{1} N_{2}} \sin \varphi
\end{aligned}
$$

where the relative phase is given by

$$
\varphi=\varphi_{1}-\varphi_{2}
$$

and evolves in time according to

$$
\dot{\varphi}=(1-R) \cos p \cdot e^{-\eta}-\omega\left(\sqrt{\frac{N_{2}}{N_{1}}}-\sqrt{\frac{N_{1}}{N_{2}}}\right) \cos \varphi .
$$




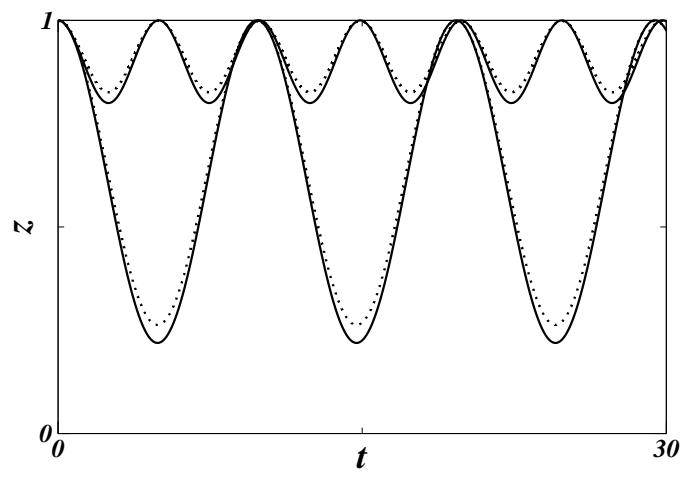

FIG. 4: Fractional imbalance $z$ as a function of time for $p=\pi$ obtained from the numerical solution (solid line) of the coupled DNLSEs for two different values of $R$, namely 1.5 (top), and 2.2 (bottom); the dotted line is the solution of Eqs. (37)-(38) for the same values of $R$, namely 1.5 (top), and 2.2 (bottom). The values of the other parameters are the ones used in Fig 2

To provide an estimate for the behaviour of $N_{1,2}$ from the system (28)-(35), we introduce the approximation $e^{-1 /(2 \alpha)} \approx 1$, which is reasonable for broad solitons. In this case, by introducing the fractional imbalance

$$
z=N_{1}-N_{2}
$$

the following equations for $z$ and $\varphi$ are obtained

$$
\begin{aligned}
\dot{z} & =-2 \omega \sqrt{1-z^{2}} \sin \varphi \\
\dot{\varphi} & =(1-R) \cos p+2 \omega \frac{z}{\sqrt{1-z^{2}}} \cos \varphi .
\end{aligned}
$$

One readily realizes that Eqs. (37)-(38) are the equations of a dimer [39, 40], but without the mass term (i.e., corresponding then to a non-interacting dimer) and with a detuning term. These equations describe the dynamics of a BEC in a double-well potential [41, 42] and analytical expressions for the quantities of interest can be found [39, 40, 42]. One of the effects of the detuning is to induce the deviations from the complete transfer of the particles from one mode to the other, which is what is numerically found. In Fig. 4, we compare the numerical results obtained from the coupled DNLSEs with the solutions of Eqs. (37)-(38); it is clear that the agreement between the two is fairly good.

\section{LINEARLY COUPLED ABLOWITZ-LADIK EQUATIONS}

The Ablowitz-Ladik equation is an integrable variant of the DNLSE [35], which in dimensionless units can be expressed as

$$
i \frac{\partial \Psi_{j}}{\partial t}=-\left(\Psi_{j-1}+\Psi_{j+1}\right)\left(1+\Lambda\left|\Psi_{j}\right|^{2}\right) .
$$

Accordingly, a system of two linearly coupled Ablowitz-Ladik equations can be written as

$$
i \frac{\partial}{\partial t}\left(\begin{array}{l}
\psi_{(1) j} \\
\psi_{(2) j}
\end{array}\right)=-\left(\begin{array}{l}
\psi_{(1) j+1}+\psi_{(1) j-1} \\
\psi_{(2) j+1}+\psi_{(2) j-1}
\end{array}\right)\left[1+\Lambda\left(\left|\psi_{(1) j}\right|^{2}+\left|\psi_{(2) j}\right|^{2}\right)\right]+\omega(t)\left(\begin{array}{l}
\psi_{(2) j} \\
\psi_{(1) j}
\end{array}\right) .
$$

Notice that this version of the vector Ablowitz-Ladik equation for $\omega=0$ constitutes the integrable vector (Manakovlike) generalization of the one-component Ablowitz-Ladik model [17]. However, it is different from other coupled models of this type with either nonlinear [43] or linear [44] coupling.

By defining $\phi_{i}$ through the unitary transformation (10), substituting in Eq. (40) and multiplying by $U^{\dagger}$, one obtains

$$
i \frac{\partial}{\partial t}\left(\begin{array}{l}
\phi_{(1) j} \\
\phi_{(2) j}
\end{array}\right)=-\left(\begin{array}{c}
\phi_{(1) j+1}+\phi_{(1) j-1} \\
\phi_{(2) j+1}+\phi_{(2) j-1}
\end{array}\right)\left[1+\Lambda\left(\left|\phi_{(1) j}\right|^{2}+\left|\phi_{(2) j}\right|^{2}\right)\right],
$$


which is in fact the same as Eq. (40) but without the Rabi term. In obtaining Eq. (41), one takes advantage of the fact that $\left|\psi_{(1) j}\right|^{2}+\left|\psi_{(2) j}\right|^{2}=\left|\phi_{(1) j}\right|^{2}+\left|\phi_{(2) j}\right|^{2}$.

Since the integrable Eq. (41) (for which soliton solutions are analytically available [17]) does not depend on $\omega$, the analysis presented in Section II is applicable and yields the same results for the dynamics of an (exact) soliton wavefunction that corresponds to all particles being initially in one of the condensates.

\section{CONCLUSIONS}

In this work we considered the soliton dynamics in a system of two discrete, linearly-coupled, nonlinear Schrödinger equations. We showed that there is a unitary transformation that can be applied to the system and has the effect of eliminating the time-dependent linear coupling terms in the case when the nonlinear coupling coefficients are equal. We showed that the solitonic solutions that describe the number of particles of a two-component Bose gas, can oscillate from one species to the other. In the case where the tunneling rates are different, although the transformation can still be made, it has no simplifying effect on the analysis. Hence, we resorted to a numerical study of the problem and found good agreement with the results obtained from an effective two-mode model. These results indicate that the efficiency of the transfer mechanism is reduced monotonically with the deviation from the equal tunneling rate limit (see also [37]). Finally, we showed that the same unitary transformation can also be applied in the analysis of a system of two linearly-coupled Ablowitz-Ladik equations, transforming the linearly coupled vector model into the well-known integrable vector Ablowitz-Ladik equation.

There are many extensions that can be considered in connection with this work. The same considerations can be applied to higher dimensions, where it is known that while continuum nonlinear Schrödinger solitons are unstable to collapse, discrete ones may be stable for sufficiently weak tunneling [15, 45]. On the other hand, one can generalize the phenomena examined herein to the case of true spinor condensates, e.g. for spin-1 (or higher) bosonic systems of ${ }^{87} \mathrm{Rb}$ or ${ }^{23} \mathrm{Na}$ which have been experimentally realized [46, 47] and are under intense theoretical investigation 48, 49, 50, 51]. Notice that using a three-mode approach for analyzing the transfer would be a particularly relevant [47] approach in that setting.

\section{Acknowledgements}

A.T gratefully acknowledges discussions with P. Sodano, A. Smerzi, L. De Sarlo, C. Fort and F. Minardi and support by the MIUR project "Quantum Noise in Mesoscopic Systems". P.G.K. gratefully acknowledges support from NSF-CAREER, NSF-DMS-0505663 and NSF-DMS-0619492. The work of D.J.F. was partially supported by the Special Research Account of the University of Athens.

[1] S. Flach and C.R. Willis, Phys. Rep. 295, 181 (1998).

[2] A. C. Scott, Nonlinear Science: Emergence and Dynamics of Coherent Structures (Oxford University Press, Oxford, 1999).

[3] D. Hennig and G. P. Tsironis, Phys. Rep. 307, 333 (1999).

[4] J. Hudock, P. G. Kevrekidis, B. A. Malomed, and D. N. Christodoulides, Phys. Rev. E 67, 056618 (2003).

[5] P. G. Kevrekidis and D. E. Pelinovsky, Proc. Roy. Soc. A 462, 2671 (2006).

[6] C. J. Myatt, E. A. Burt, R. W. Ghrist, E. A. Cornell, and C. E. Wieman, Phys. Rev. Lett. 78, 586 (1997).

[7] D. S. Hall, M. R. Matthews, J. R. Ensher, C. E. Wieman, and E. A. Cornell, Phys. Rev. Lett. 81, 1539 (1998).

[8] K. M. Mertes, J. W. Merrill, R. Carretero-González, D. J. Frantzeskakis, P. G. Kevrekidis, and D. S. Hall, Phys. Rev. Lett. 99, 190402 (2007).

[9] D. M. Stamper-Kurn, M. R. Andrews, A. P. Chikkatur, S. Inouye, H.-J. Miesner, J. Stenger, and W. Ketterle, Phys. Rev. Lett. 80, 2027 (1998); M.-S. Chang, C. D. Hamley, M. D. Barrett, J. A. Sauer, K. M. Fortier, W. Zhang, L. You, and M. S. Chapman, Phys. Rev. Lett. 92, 140403 (2004).

[10] G. Modugno, G. Ferrari, G. Roati, R.J. Brecha, A. Simoni, and M. Inguscio, Science 294, 1320 (2001).

[11] M. Mudrich, S. Kraft, K. Singer, R. Grimm, A. Mosk, and M. Weidemüller, Phys.Rev. Lett. 88, 253001 (2002).

[12] J. Catani, L. De Sarlo, G. Barontini, F. Minardi, and M. Inguscio, Phys. Rev. A 77, 011603(R) (2008); G. Thalhammer, G. Barontini, L. De Sarlo, J. Catani, F. Minardi, and M. Inguscio, Phys. Rev. Lett. 100, 210402 (2008).

[13] O. Morsch and M. Oberthaler, Rev. Mod. Phys. 78, 179 (2006).

[14] A. Trombettoni and A. Smerzi, Phys. Rev. Lett. 86, 2353 (2001).

[15] P. G. Kevrekidis, K. Ø. Rasmussen, and A. R. Bishop, Int. J. Mod. B 15, 2833 (2001). 
[16] A. Smerzi and A. Trombettoni, "Optical Lattices: Theory", in Emergent Nonlinear Phenomena in Bose-Einstein Condensates: Theory and Experiment, edited by P. G. Kevrekidis, D. J. Frantzeskakis, and R. Carretero-González, (Springer, Berlin, 2008), pp. 247-265.

[17] M. J. Ablowitz, B. Prinari, and A. D. Trubatch, Discrete and Continuous Nonlinear Schrödinger Systems, (Cambridge University Press, Cambridge, 2004).

[18] H. S. Eisenberg, Y. Silberberg, R. Morandotti, A. R. Boyd, and J. S. Aitchison, Phys. Rev. Lett. 81, 3383 (1998).

[19] B. A. Malomed and M. I. Weinstein. Phys. Lett. A 220, 91 (1996).

[20] A. B. Aceves, C. De Angelis, T. Peschel, R. Muschall, F. Lederer, S. Trillo, and S. Wabnitz, Phys. Rev. E 53, 1172 (1996).

[21] D. B. Duncan, J. C. Eilbeck, H. Feddersen, and J. A. D. Wattis, Physica D 68, 1 (1993).

[22] S. Flach and K. Kladko, Physica D 127, 61 (1999).

[23] J. Gomez-Gardenes, L. M. Floria, M. Peyrard, and A. R. Bishop, Chaos 14, 1130 (2004).

[24] T. R. Melvin, A. R. Champneys, P. G. Kevrekidis, and J. Cuevas, Phys. Rev. Lett. 97, 124101 (2006).

[25] O. F. Oxtoby and I. V. Barashenkov, Phys. Rev. E 76, 036603 (2007).

[26] O. Mandel, M. Greiner, A. Widera, T. Rom, T. W. Hánsch, and I. Bloch, Phys. Rev. Lett. 91, 010407 (2003).

[27] D. M. Stamper-Kurn and W. Ketterle, in Coherent Atomic Matter Waves, Les Houches Summer School Session LXXII, edited by R. Kaiser, C. Westbrook, and F. David (Springer, New York, 2001), pp. 137-217 (arXiv:cond-mat/0005001).

[28] D. S. Hall, in Emergent Nonlinear Phenomena in Bose-Einstein Condensates: Theory and Experiment, edited by P. G. Kevrekidis, D. J. Frantzeskakis, and R. Carretero-González, (Springer, Berlin, 2008), pp. 307-327.

[29] A. Smerzi, A. Trombettoni, T. Lopez-Arias, C. Fort, P. Maddaloni, F. Minardi, and M. Inguscio, Eur. Phys. J. B 31, 457 (2003).

[30] J. Williams, R. Walser, J. Cooper, E. A. Cornell, and M. Holland, Phys. Rev. A 61, 033612 (2000).

[31] Notice that for a Bose-Bose mixture composed by different bosonic species one has $K_{1} \neq K_{2}$, with $K_{1}$ larger than $K_{2}$ if $m_{2}<m_{1}\left(m_{\alpha}\right.$ being the atomic mass of the bosonic species $\left.\alpha\right)$; however, for a Bose-Bose mixture of different species, the Rabi tunneling can not be implemented.

[32] B. Deconinck, P. G. Kevrekidis, H. E. Nistazakis, and D. J. Frantzeskakis, Phys. Rev. A 70, 063605 (2004).

[33] M.V. Tratnik and J.E. Sipe, Phys. Rev. A 38, 2011 (1988).

[34] R. M. Bradley, B. Deconinck and J. N. Kutz, J. Phys. A 38, 1901 (2005).

[35] M. J. Ablowitz and J. F. Ladik, J. Math. Phys. 17, 1011 (1976).

[36] S. V. Manakov, Zh. Eksp. Teor. Fiz. 65, 505 (1973) [Sov. Phys. JETP 38, 248 (1974)].

[37] H. Nistazakis, Z. Rapti, D. J. Frantzeskakis, P. G. Kevrekidis, P. Sodano, and A. Trombettoni, arXiv:0805.0189 (Phys. Rev. A, in press).

[38] C. Paré and M. Florjańczyk, Phys. Rev. A 41, 6287 (1990); B. A. Malomed, I. M. Skinner, P. L. Chu, and G. D. Peng, Phys. Rev. E 53, 4084 (1996); A. I. Maimistov, Sov. J. Quantum Electron. 21, 687 (1991).

[39] V. M. Kenkre and D. K. Campbell, Phys. Rev. B 34, 4959 (1986).

[40] V. M. Kenkre and G. P. Tsironis, Phys. Rev. B 35, 1473 (1987).

[41] A. Smerzi, S. Fantoni, S. Giovanazzi, and S. R. Shenoy, Phys. Rev. Lett. 79, 4950 (1997).

[42] S. Raghavan, A. Smerzi, S. Fantoni, and S. R. Shenoy, Phys. Rev. A 59, 620 (1999).

[43] A. Bülow, D. Hennig and H. Gabriel, Phys. Rev. E 59, 2380 (1999).

[44] B.A. Malomed and J. Yang, Phys. Lett. A 302, 163 (2002).

[45] P.G. Kevrekidis, K.Ø. Rasmussen and A.R. Bishop, Phys. Rev. E 61, 2006 (2000).

[46] J. M. Higbie, L. E. Sadler, S. Inouye, A. P. Chikkatur, S. R. Leslie, K. L. Moore, V. Savalli, and D. M. Stamper-Kurn, Phys. Rev. Lett. 95, 050401 (2005).

[47] M.-S. Chang, Q. S. Qin, W. X. Zhang, L. You, and M. S. Chapman, Nature Phys. 1, 111 (2005).

[48] H. Saito and M. Ueda, Phys. Rev. A 72, 023610 (2005).

[49] W. Zhang, Ö. E. Müstecaplioglu, and L. You, Phys. Rev. A 75, 043601 (2007).

[50] B.J. Dabrowska-Wüster, E. A. Ostrovskaya, T. J. Alexander, and Yu. S. Kivshar, Phys. Rev. A 75, 023617 (2007).

[51] H. E. Nistazakis, D. J. Frantzeskakis, P. G. Kevrekidis, B. A. Malomed, R. Carretero-González, and A. R. Bishop, Phys. Rev. A 76, 063603 (2007); H. E. Nistazakis, D. J. Frantzeskakis, P. G. Kevrekidis, B. A. Malomed, and R. CarreteroGonzález, Phys. Rev. A 77, 033612 (2008). 